\title{
LES SOURIRES ET LES LARMES. OBSERVATIONS EN MARGE DE QUELQUES TEXTES HAGIOGRAPHIQUES MUSULMANS
}

\author{
Giovanna CALASSO \\ Università «La Sapienza» - Roma
}

La littérature hagiographique musulmane offre un terrain privilégié d'exploration en ce qui concerne les modalités de vivre et de penser l'expérience religieuse de l'Islam à un niveau qui ne coïncide pas avec celui de ses manifestations légales, celles qui définissent officiellement l'appartenance d'un individu à la communauté musulmane, mais qui manifestement ne sont pas les seules modalités de cette appartenance. Plus que du rapport entre des sujets et Dieu, il s'agit dans ces récits du rapport qui a lié l'individu narrateur et les saints, ainsi que de la transmission du témoignage d'une pluralité d'autres individus qui ont partagé ce même type d'expérience. De ces textes nous pouvons tirer quelques éléments qui concernent la dimension personnelle, individuelle de la religion, les émotions qu'elle produit, la façon dont elle est pensée et agie; ils nous offrent, en même temps, un témoignage aussi digne d'intérêt en ce qui concerne les modalités médiévales de l'écriture des sentiments et des émotions religieuses.

L'ouvrage d'Ibn Qunfudh, Uns al-faqir wa-'izz al-haqir, de la moitié du XIVe siècle, va être le texte guide, qu'on comparera parfois avec le Rüh al-quds d'Ibn 'Arabī, d'une première reconnaissance dans ce domaine. Précédé par une partie introductive qui définit les prérogatives des wali et les classifie en differents catégories, l'ouvrage traite aussi la question des miracles des saints, et notamment de la possibilité qu'ils se réalisent après la mort du saint. Dans la deuxième partie de l'ouvrage, la figure d'Abū Madyan et des personnages qui l'ont entouré -maîtres, disciples, confrères, compagnons- devient le centre d'intérêt d'Ibn Qunfudh, et les mémoires liées à la personne de son grand-père Yūsuf $b$. Ya 'qūb al-Malārī, ainsi qu'à celle de son arrière-grand-père Ya'qūb b. 'Imrān alBūyūsufî, soufis eux aussi, établissent un trait d'union familial entre l'auteur et le maître Abū Madyan'1. La troisième partie est constituée par le récit des pérégrinations de l'auteur, à partir de la ville de Constantine, à la recherche d'hommes saints et d'ordres saints jusque dans la région du Maroc méridional, sur la côte de l'Océan. Écriture hagiographique et récit de voyage se conjuguent

\footnotetext{
${ }^{1}$ Ibn Qunfudh, Uns al-faqïr wa-'izz al-haquir, texte arabe établi et publié par M. El Fasi et A. Faure, Rabat, 1965, 40-42. Le lien avec Abū Madyan s'établit par l'intermédiaire de son disciple Abū Mas'ūd b. 'Arīf.
} 
ici en produisant une riḥla ziyāriyy $a^{2}$, même si la dimension «voyage» n'y est qu'apparente: il n'y a pas de vrais «lieux» dans l'Uns al-faqir en dehors des personnes des saints, et le temps n'est dans le récit que l'espace de la rencontre avec eux: qu'il s'agisse d'Abū Madyan, de ses maitres ou de ses disciples, donc de rencontres qui ont eu lieu dans le passé et dont Ibn Qunfudh n'a pu que recueillir les témoignages, ou de rencontres qu'il a vécues directement.

Lorsqu'on se met à la recherche, dans ce texte, de traces qui se réfèrent à l'expérience religieuse individuelle, ce qui apparaît comme une constante, c'est que ces traces passent toujours par le langage du corps. Les sens ont beaucoup à voir avec la transcription de ce type d'expérience: la vue et le toucher en premier lieu, parfois le goût et l'odorat, alors que l'ouïe qui joue un rôle de premier plan dans la transmission «traditionnelle» du savoir religieux, est souvent rendue superflue dans ce contexte par une transmission de l'enseignement qui a lieu la plupart des fois dans le silence, grâce à une expérience de contact direct. Loin d'être exclu des états spirituels, le corps y participe de façon intégrale: «Je dormis toute la nuit à côté de notre maître Abū Mas'ūd dans son lieu de retraite, et je sentis chacun de ses cheveux invoquer Dieu de façon éloquente» ${ }^{3}$. C'est le témoignage de Ya'qūb b. 'Imrān al-Būyūsufî, arrière-grand-père d'Ibn Qunfudh; un siècle et demi avant lui, le shaykh Abū 'Abdallāh b. Qassūm, dont Ibn 'Arabī fut le compagnon pendant près de dix-sept ans et qui lisait le Coran presque sans interruption, le plaçant sur ses genoux, suivant les lettres avec son doigt et le psalmodiant, repondit à Ibn 'Arabī «qu'il faisait cela afin que chacun de ses membres pût tirer profit de cette lecture» ${ }^{4}$.

Le corps est soumis autant que l'âme aux épreuves les plus dures: «Son zèle et ses efforts étaient tels qu'il préparait des baguettes pour se fustiger les jambes lorsqu'elles étaient fatiguées... et continuait à les frapper jusqu'à ce que les baguettes fussent cassées», racontait Ibn 'Arabī de son oncle maternel Abū Muslim al-Khakwānī ${ }^{5}$, mais les exemples de ce genre pourraient se multiplier à l'infini. Toutefois, ce qu'on veut surtout souligner ici, c'est que le corps nous apparaît, dans ces textes, comme étant un véhicule de connaissances spirituelles.

Voilà le témoignage du savant Ibn Riḍwān qui raconte, se référant au grandpère d'Ibn Qunfudh:

«Lorsque nous allâmes lui rendre visite et qu'il nous offrit à manger, il mit juste à côté de moi le mets que moi seul je désirais, en souriant ( $w a$ -

2 Voir Sebti, A., «Hagiographie du voyage au Maroc médiéval», Al-Qantara XIII (1992), 167-179.

3 Ibn Qunfudh, op. cit., 41-42.

${ }^{4}$ Ibn 'Arabī, Les soufis d'Andalousie (Ruḥ al-Quds et al-Durrat al-făhira), Introduction et traduction par R. W. J. Austin, Paris, 1979, 91.

${ }^{5}$ Ibid., 122. 
huwa yatabassamu). Et j'y vis ce que je n'aurais jamais cru pouvoir connaître» 6 .

Un mets que l'on aime goûter et qui est offert précisement dans le but de faire plaisir à celui qui le reçoit, un sourire, et voilà que s'établit une intimité qui permettra d'arriver à la connaissance spirituelle, à la compréhension. Une expérience pareille est racontée par Ibn Qunfudh. Il s'agit de sa rencontre avec Ibn 'Āshir: «La première fois que je le rencontrai, il se déroba, mais je l'arrêtai avec ma main et le secouai. Alors il sourit (tabassama), s'arrêta, me posa des questions sur mon âme, et pria pour moi $\left(d a^{\prime} \bar{a} l \bar{l}\right)$ avec bienveillance». À l'occasion d'une autre rencontre, Ibn Qunfudh lui ayant demandé quelque chose à manger:

«Il me demanda pardon parce qu'il n'avait rien à me donner, puis il me dit: 'Attends-moi' et il entra dans sa maison. Peu après il en sortit en apportant des graines de figues sèches, les tenant dans sa main droite et les recouvrant de sa main gauche, il me les offrit et rit avec moi (wa-dahika ma $i$ ). Les autres furent émerveillés de la tendresse et de la gaité qu'il montrait dans son comportement envers moi, puisque d'habitude il n'était expansif avec personne. Ceci fut pour moi un grand honneur, et personne ne peut comprendre sa valeur, si ce n'est celui qui a essayé de le conquérir»?.

Les gestes, décrits d'une façon très directe, remplissent la scène: le sourire, ou le rire et le geste d'offrir un aliment, même pauvre, mais que le saint donne de ses propres mains, sont les signes d'un choix, d'un privilège accordé par le saint au disciple, par lequel une communication spirituelle, personnelle et exclusive est rendue possible.

En lisant Ibn Qunfudh, mais aussi d'autres textes hagiographiques, on s'aperçoit que l'offre d'un aliment ou d'un mets préparé par le saint de ses propres mains, ou que le toucher de sa main rend exquis, fait partie des topo $\ddot{\imath}$ narratifs de ces textes.

Dans le Bustān, oeuvre de l'hagiographe de Tilimsān Ibn Maryam, on en trouve un exemple tout particulièrement riche en détails, dans la biographie de l'ex-juge de Seville Abū 'Abdallāh al-Shūdhī al-Ishbīî, plus connu comme Sīdī 'l-Halwī, qui, ayant abandonné sa profession et s'étant transféré au Maghreb, dissimulait sa sainteté sous l'apparence d'un vendeur ambulant de sucreries; sur lui, voici le récit d'Ibn al-Marāt, transmis tel quel par Ibn Maryam:

${ }^{6}$ Ibn Qunfudh, op. cit., 44-45.

${ }^{7}$ Ibid., 9. 
«Je vins de Murcie, pour voir une tante paternelle que j'avais à Tlemcen... Un jour que je me promenais dans les rues, je rencontrai ce cheikh tenant à la main un plateau en bois, rempli de pâtes sucrées qu'il vendait aux petits enfants, et je crus reconnaitre en lui les traits caracteristiques des soufis... Nous étions alors dans le mois de Ramaḍan. Lorsque le jour de la rupture du jeûne fut arrivé, j'achetai de la semoule et du miel et je dis à ma tante: "Faites-moi quelques gateaux meshehda pour les repas de la rupture du jeûne, que je ferai en compagnie d'un saint homme". Elle fit selon mon désir...». Mais le saint est introuvable parmi la foule du jour de fête; alors Ibn al-Marāt adresse une prière a Dieu et voilà le saint qui apparaît: «Il me regarda et me dit: "Votre tante a-t-elle préparé les meshehda?" Oui, sīdī, répondis-je. "Eh bien, ajouta-t-il, retirons nous en quelque lieu pour manger les meshehda que voici, puis nous irons à la maison de votre tante". Lorsque nous fûmes hors de la mosquée, il tira de dessous ses habits un plat recouvert d'un foulard propre, et les plus belles meshehda qu'on n'ait jamais vues et que jamais femme au monde n'ait si bien réussies sous le rapport de la cuisson, de la préparation et de l'abondance des condiments gras, s'offrirent à ma vue. Après en avoir mangé, nous nous rendîmes chez ma tante qui nous présenta les meshehda qu'elle avait préparées, mais nous ne le trouvâmes comparables en rien aux premières et c'est à peine si nous y touchâmes». ${ }^{8}$

Ce récit, détaillé et subtilement mysogine, souligne en détail toutes les qualités des gâteaux du saint, comme s'il les avait préparés de ses propres mains, en faisant ressortir la perfection de son art en ce qui concerne les règles (ahquām) de la cuisson, de l'exécution, ainsi que du dosage des ingrédients. Presqu'une parabole, qui précède le récit de la fréquentation qui va s'instaurer entre les deux, pendant laquelle le saint offrira au disciple la nourriture spirituelle de son enseignement ${ }^{9}$.

La sainte Mu'mina Tilimsāniyya que Ibn Qundufh rencontre à Fès, la vieille ascète qu'on voyait circuler la tête couverte d'un vieux tissu en lambeaux dont quelques cheveux blancs s'échappaient sur le front, n'était pas tendre avec elle même. Elle conduisait une vie d'ascétisme et dévotion, sans rien accepter des autres; et pendant les mois de Rajab, Sha'bān et Ramaḍān elle se retirait dans l'isolement le plus complet. Un jour, lorsqu'Ibn Qunfudh lui demande si elle a besoin de quelque chose, elle répond:

\footnotetext{
8 Ibn Maryam, El Bostan ou Jardin des biographies des saints et savants de Tlemcen, traduit et annoté par F. Provenzali, Alger, 1910, 74-75 (al-Bustān fi dhikr al-awliyā' 'wa-'l-'ulamā' biTilimsān, ed. Muhammad b. Abī Shanab, al-Jazā’ir 1336/1908, 68-69).

${ }^{9}$ Ibid., 75-76.
} 
«"Juste d'une arête pour nettoyer mes dents". J'allai à sa recherche et je trouvai, à proximité d'un four, un sac plain d'arêtes - continue Ibn Qunfudh. Je me rejouis de les avoir trouvées et je revins chez elle. "Où les as-tu trouvées?" me dit-elle; à ma réponse, elle demanda: "As-tu reçu la permission de les prendre? Ramène-les". J'allai donc chercher le propriétaire des arêtes pour lui demander la permission, qu'il m'accorda promptement, et revenu chez elle pour lui rapporter la chose, elle me dit: “Ce n'est pas ce dont j'avais besoin. Maintenant va-t-en et que Dieu te protège!". Je m'en allai et je compris son but, qui était celui de me faire saisir les règles de conduite même les plus insignifiantes et de m'en faire tirer profit». «Une fois, raconte encore Ibn Qunfudh, je lui apportai en cadeau des biscuits, en la priant de les accepter, ce qu'elle fit; et chaque fois que j'allais chez elle pour lui rendre visite, elle m'offrait un biscuit, sans que je puisse comprendre d'où il venait. Un jour, après m'en avoir offert un comme d'habitude, elle sourit en me disant: "Ceci est le dernier des biscuits que tu m'avais donnés; quant à moi, je me suis nourrie de ta satisfaction"» ${ }^{10}$.

Il n'y a que des «choses» dans ce récit, et il s'agit de choses qui sont pour la plupart liées à l'alimentation: un sac d'arêtes, un four, des biscuits. C'est à travers la nourriture, dont la pieuse Mu'mina s'abstient rigoureusement, mais qu'elle offre à son disciple se nourrisant «de sa satisfaction», que devient possible un apprentissage informel, graduel — les biscuits qu'il lui avait offerts, elles les lui rend un à chaque visite - un apprentissage qui n'est pas reçu par transmission auditive, mais par l'intermédiaire d'un contact direct avec la sainte et des humbles gestes qu'elle lui impose en les utilisant comme des métaphores; le sourire de la sainte est un signe d'entente et un véhicule d'enseignement.

Parfois on a même l'impression que le sourire du saint puisse exercer sur le disciple une sorte de ravissement qui le soustrait à la perception de la douleur physique. Ainsi le shaykh al-Kūmī, après avoir parlé à Ibn 'Arabī des vertus et des miracles du maître Abū Madyan, le regarda soudainement et sourit: «Puis, il éperonna son cheval et pressa l'allure et je hâtais le pas pour me maintenir à sa hauteur. Finalement il s'arrêta et me dit: "Regarde ce que tu as laissé derrière toi!". En me retournant, je vis que tout le chemin parcouru n'était que ronces qui arrivaient à mi-corps. Il me dit alors de regarder mes pieds et mes vêtements. Je baissai les yeux, mais ne remarquai pas la moindre égratignure. "Ceci est dû à la grâce spirituelle (baraka) attachée à notre conversation sur Abū Madyan", dit-i ${ }^{11} \gg$. Mais n'est-ce pas le sourire du saint qui a pu préserver, presque comme un baume protecteur, le corps du disciple Ibn 'Arabī?

\footnotetext{
${ }^{10}$ Ibn Qunfudh, op. cit., 81.

1 Ibn 'Arabī, op. cit., 71.
} 
La rị̣la d'Ibn Qunfudh nous donne aussi un témoignage de l'émotion produite par le passage, expérimenté par l'auteur, d'un savoir acquis par transmission auditive, à un type de savoir directement acquis par des canaux qui impliquent la participation d'autres sens que l'ouie. C'est dans cette direction qu'on peut trouver d'autres fragments de la dimension individuelle de son expérience religieuse.

Nous reproduisons ci-dessous le récit qu'Ibn Qunfudh nous a laissé de l'assemblée des fuqarā' à laquelle il participa personnellement en 769 , pour laisser que ce soient ses mots, passant progressivement de la description des choses qu'il a vues à la réflexion sur le fait de «voir de ses propres yeux», à nous transmettre l'impact émotionnel de cette expérience:

«Je fus présent, raconte Ibn Qunfudh, avec un groupe de ces confréries (tawä'if), dont la plupart demeurait dans l'extrême sud du Maroc, sur les rivages de l'Océan, au rassemblement des fuqarā' dans la région des Dukkāla, entre la ville de Asfi et celle de Tayțanfațar, nom qui signifie 'la source froide'. C'est une plaine qui mesure en largeur quatre journées de marche et autant en longueur. Au temps de mon arrivée dans cette région, la quantité de terrain cultivable s'élévait à dix-mille zawāj et le nombre d'animaux était en excédent. Le rassemblement eu lieu pendant le mois béni, heureux et resplendissant de $R a b i$ 'al-awwal, en 769 , et d'innombrables fuḍalā' y participèrent. J'y rencontrai les saints et les savants les plus éminents; ils étaient si nombreux que mes yeux en restèrent effrayés. Ce qui prouve à quel point ils étaient nombreux, est le fait que certains de nos compagnons des Dukkāla qui désiraient ardemment leur $d u^{\prime} \bar{a}^{\prime}$, achetèrent pour eux du raisin pour trente dinars, mais ce ne fut pas suffisant. Et bien, ce jour là, je me renseignai sur le prix du raisin et j'appris qu'un panier coûtait un grand dirham, c'est à dire un tiers du nouveau dirham tunisien. Il y avait là des malades affectés d'une invalidité permanente, des paralytiques et autres. Je les ai vus se presser dans les cercles de $d h i k r$, implorer humblement en désirant ardemment la guérison du corps, comme s'ils imploraient de la nourriture. Et voilà quelqu'un qui se lève, prend le malade par la main et le malade s'en va déjà guéri. Voilà un autre qui touche le malade d'un pan de son vêtement et le malade se lève comme libéré de chaines, et celui qui a opéré le miracle se mélange à la foule et disparaît. J'avais entendu dire tout ceci, mais maintenant je l'ai vu, par Dieu, de mes propres yeux! (wa-hādhā kuntu asma'uhu hạtā ra'aytuhu wallāhi 'iyānan)».

Après avoir décrit le miracle de la guérison d'un adolescent dont les jambes étaient atteintes par un spasme, le récit se termine par ces mots: 
«Je vis dans cette assemblée des choses étranges et merveilleuses (gharā'ib $\left.w a-{ }^{\prime} a j \bar{a} ' i b\right)$, qui ne seront jamais plus revues par la suite car les conditions ont changé» ${ }^{12}$.

Ibn Qunfudh mesure d'abord et quantifie, à la manière des voyageurs ${ }^{13}$ les dimensions de la plaine où a lieu l'assemblée des fuqarā', les terrains cultivés, les animaux, le prix du raisin. Puis, ce sont les gestes, la souffrance et la joie, l'impatience du contact et la dévotion des gens qui font l'objet de sa description, alors que la scène est remplie par la présence silencieuse du pouvoir des saints. Hors l'effroi suscité dans son esprit par la présence de cette multitude des șălihūun, rien d'explicite ne filtre de ses propres sentiments et émotions. Tout se passe comme si, en écrivant, il ne lui était permis que de se placer comme témoin d'autres individus sans mettre explicitement en jeu son individualité. Il n'y a qu'une chose qu'Ibn Qunfudh ne peut s'empêcher de dire à haute voix: la distance qui sépare un savoir réçu par transmission auditive d'un savoir acquis par ses propres yeux. Mais ce n'est que l'interjection wallāhi qui signale qu'il se sent impliqué à la première personne, tandis que quelques lignes après, l'expression stéréotypée 'ajā'ib wa-gharā'ib, utilisée par Ibn Qunfudh pour faire allusion à toutes les choses bouleversantes dont il a été témoin, nous dit qu'il ne veut pas —ou qu'il ne peut pas- aller au delà pour communiquer au lecteur sa propre expérience personnelle.

De même, en se référant à sa rencontre avec le maître de la tậyfa des Hujjāj, il s'était borné à dire: «Je vis, lorsque j'étais avec ce maitre, des choses étranges et merveilleuses» ${ }^{14}$. Et plus loin, résumant ses impressions après avoir passé la nuit en compagnie du saint Abū 'l-Ḥasan Yūsuf al-Ṣanhājī: «Ce fut une nuit si merveilleuse qu'un livre entier ne suffirait pas si je voulais la décrire en détail» ${ }^{15}$. Presque les mêmes mots que ceux qu'aurait dit son grand-père, soufi lui aussi, à propos d'une nuit passée à la mosquée en compagnie du maître Abū Hādī: «Je jure sur Dieu que cette nuit-là, nous vîmes des choses et des faits extraordinaires qu'il n'est pas possible de décrire» ${ }^{16}$. Frontières invisibles, mais nettement tracées, et partagées à travers les générations, marquent les limites entre ce qui est dicible et ce qui ne l'est pas, entre ce qui peut être écrit et ce qu'il n'est pas possible de confier à l'écriture.

${ }^{12}$ Ibn Qunfudh, op. cit., 71-72.

${ }^{13}$ Sur ce thème, voir Calasso, G., «Les tâches du voyageur: décrire, mesurer, compter, chez Ibn Jubayr, Nāṣer-e Khosrow et Ibn Baț̣ūṭ», Rivista degli Studi Orientali, LXXIII, 1999 (à paraître).

${ }^{14}$ Ibn Qunfudh, op. cit., 64.

15 Ibid., 72

${ }^{16}$ Ibid., 52. 
À propos du maître Abū 'l-Ḥasan al-Ṣanhājī', Ibn Qunfudh nous dit pourtant beaucoup plus:

«Ce fut pour moi un grand honneur de le rencontrer et de tirer profit de sa baraka et de sa prière (ightināmi li-barakatihi wa-du'a'ihi). Son corps était extrèmement gras et des larmes coulaient incessantes de ses yeux. Chaque fois qu'il écoutait un verset du Coran ou un hadith du Prophète ou un morceau de poésie ou la parole, d'un maître soufî il se couvrait les yeux des paumes de la main et commançait à pleurer si fort qu'on craignait pour lui. 'Ce saint mourra de pleurs!' je dis à un des fuḍalā' qui étaient présents... Et quand il pleurait ainsi, ceux qui participaient à la réunion étaient saisis de peur et les larmes coulaient des yeux de tous».

Puis, de l'observation oculaire «externe», il passe au récit de son expérience personnelle:

«Quand al-Ṣanhājī me voyait, il s'arrêtait, m'embrassait, caressant mes épaules de sa main bénie, et pleurait, pleurait et ne me quittait qu'au bout d'une heure. Son aspect qui inspirait une crainte révérentielle, et l'intensité de son état spirituel me faisaient frissonner» ${ }^{17}$.

Le corps du saint, son aspect physique ne sont pas ignorés dans ce récit, et, encore une fois, c'est le corps qui se fait l'interprète de formes d'émotion spirituelle: les frissons ressentis par Ibn Qunfudh, nous apparaissent dans ce contexte, à la différence de la crainte et des larmes, comme un petit détail nonconventionnel, qui laisse filtrer une sensation individuelle. On pourrait comparer ce récit à celui d'Ibn 'Arabī, lorsqu'il décrit ses réactions en présence du shaykh al-Kūmī: «Il s'adossa au mihrāb, me tendit le livre et me dit: "Lis". La crainte révérentielle qu'il m'inspirait était si intense que je ne pus assembler deux mots, et le livre me tomba des mains» ${ }^{18}$.

Dans les écrits des auteurs musulmans médiévaux les larmes se présentent en effet comme la manifestation somatique par excellence de l'émotion religieuse, une manifestation qu'on dirait obligée, voire conventionnelle, dans ses formes individuelles aussi bien que collectives ${ }^{19}$. Les larmes lavent, purifient, et laissent

17 Ibid., 72

18 Ibn 'Arabī, op. cit., 71.

19 Sur le thème des larmes comme manifestation somatique privilégiée qui prélude au phénomène de la conversion, dans un contexte chrétien, voir Cohen, E., «Fire and Tears: the 
en même temps apparaître un état intérieur, en le rendant visible par le langage du corps. Le saint pleure, pleurent ses disciples en le voyant pleurer, pleurent les pélerins qui s'entassent fièvreusement pour entrer dans la $\mathrm{Ka}$ 'ba, ainsi que les gens qui se bousculent pour pouvoir embrasser le tombeau du saint, pleure le prédicateur, ému par ses propres virtuosités verbales, pleure son assistance sanglotant sans retenue, pleure le converti qui a choisi de se dépouiller du monde pour s'adonner à Dieu, et on n'a jamais plus vu rire certains d'entre eux à partir de leur repentir. La littérature musulmane médiévale, hagiographique ou non, présente un large éventail d'exemples de ce genre ${ }^{20}$.

«Ce ne sont pas seulement les pleurs - écrivait Marcel Mauss - mais toutes sortes d'expressions orales des sentiments, qui sont essentiellement, non pas des phénomènes exclusivement psychologiques, mais des phénomènes sociaux, marqués éminemment du signe de la non-spontanéité et de l'obligation la plus parfaite $^{21} \gg$. Les modèles culturels ${ }^{22}$ agissent donc aussi dans des domaines comme celui des sentiments et des émotions, jadis considérés comme faisant partie d'une dimension de l'expérience humaine se soustrayant à tout genre de contrainte; ils en révèlent la présence autant dans leurs modalités de manifestation que dans les modalités de leur description, même si, en nous référant à une société du passé, nous ne pouvons énoncer cette distinction qu'au niveau théorique.

Mais si à nos yeux, les larmes du saint, ainsi que celles qu'il suscite chez ses disciples, appartiennent aux manifestations publiques conventionnelles de l'émotion, pour les maîtres soufis elle ne sont que l'expression d'un niveau plus élevé de connaissance, une manifestation somatique de contrition qui est liée à

physical Manifestations of Conversion», dans Conversioni nel Mediterraneo, a cura di A. Foa, e L. Scaraffia, Dimensioni e problemi della ricerca storica 2 (1996), 247-253. Voir aussi Christian Jr., W., «Provoked Religious Weeping», dans Davis, J. (ed.), Religious Organisation and Religious Experience, London, 1982, 97-114.

${ }^{20}$ Pour ce qui est des pèlerins et des prédicateurs, je me réfère en particulier à la Rihla de Ibn Jubayr: voir Calasso, G., «La dimension religieuse individuelle dans les textes musulmans médiévaux, entre hagiographie et littérature de voyage: les larmes, les émotions, l'expérience», Studia Islamica 91 (2000) (à paraitre). L'ouvrage de Tādilī, al-Tashawwuf ilā rijäl al-tașawwuf, offre, en revanche, de nombreux exemples en ce qui concerne les larmes en tant que marques somatiques de la conversion «interne».

21 Mauss, M., «L'expression obligatoire des sentiments» (1921), dans Oeuvres, III. Paris, 1969, 269. L'analyse de Mauss se référait à des contextes collectifs formalisés et ritualisés. La possibilité d'identifier des règles semblables restait ouverte, même dans le comportement quotidien et individuel. Restait également ouverte la question de savoir comment circonscrire le floux émotionnel, qui, selon Mauss, se prête peu à l'analyse. Sur ces thèmes, de Mauss à l'anthropologie interprétative, à l'anthropologie de l'expérience jusqu'à celle des émotions, voir De Matteis, S., «Esperienza, sentimenti, emozioni», La Ricerca folklorica, n. 35, 1997 (Antropologia dell'interiorità), 67-79. Voir aussi Lutz, C.-White, G., «The Anthropology of Emotions», Annual Review of Anthropology, 16 (1986), 405-436.

${ }^{22}$ Sur le fonctionnement des modèles culturels, voir Geertz, C., Interpretation of Cultures, New York, 1973, 89 suiv. 
une dimension rationnelle et non pas à un trouble de nature émotionnelle, étant donné que la crainte de Dieu n'est pas un signe de faiblesse, mais d'une excellence de la foi qui se manifeste chez les «gens doués d'intelligence» ${ }^{23}$. En plus, les larmes des saints peuvent aussi avoir des vertus qui ne sont pas seulement spirituelles, mais qui peuvent être perçues et appréciées par les sens: «Lorsqu'il pleurait, écrivait Ibn 'Arabī se référant au shaykh Abū Ahmad asSalawī, je recueillais les larmes qui tombaient sur le sol et m'en frottais le visage, car je trouvais qu'elles sentaient bon le musc» ${ }^{24}$.

Le contact avec le saint implique aussi la réception de deux éléments fondamentaux: la baraka et la $d u^{\prime}{ }^{a}$ ', qui son conçues comme une sorte de butin dont il faut chercher à s'emparer, mais dont les modalités de transfert sont différentes: la baraka s'écoule, pour ainsi dire, naturellement du saint et il suffit du contact pour en bénéficier; par contre la $d u$ ' 'a', invocation qui peut s'exprimer autant dans la forme du discours intérieur que dans celle d'enoncé verbal formulé25, c'est un don dont le saint fait parfois bénéficier spontanément ceux qui l'entourent, mais qu'il est plus souvent nécessaire de demander. Ainsi dans le récit suivant:

«Dans la petite ville de Sefrou, dans les faubourgs de Fès, je rencontrai un inconnu. L'occasion de le voir s'était présentée parce que j'entrai dans la mosquée un vendredi - toujours à la recherche d'hommes vertueux, selon mon habitudeà une heure qui n'était pas celle de la prière. La mosquée était vide. J'ouvris la porte d'une petite cellule près du mihrāb et vis un homme d'une maigreur extrême; je le saluai et il me rendit mon salut. Je lui demandai comment il allait, et, plusieurs fois, d'où il venait; mais il resta silencieux. Alors je restai assis pendant une heure à le regarder et j'en reçus un enseignement. Je lui demandai ensuite de prier pour moi, mais je ne l'entendis pas prononcer de mots et je ne sais pas si, à l'intérieur de lui, il avait prié pour moi ou pas. Je sortis de la mosquée et dans mon âme il y avait quelque chose (wa-fi nafsī shay')» ${ }^{26}$.

L'expérience du contact avec le saint est perçue cette fois-ci au niveau du nafs: mais il n'y a pas de langage, paraitt-il, qui permet d'en décrire les effets de la même façon qu' au niveau du corps. Ce qui se produit à l'intérieur du nafs reste indéfini (shay').

\footnotetext{
${ }^{23}$ Voir Ibn 'Arabī, op. cit., 97-98, n. 4 (je rapporte les mots d'Austin).

24 Ibid., 141.

25 Voir Calasso, G., «La dimension religieuse», cit., 44-45.

${ }^{26}$ Ibn Qunfudh, op. cit., 85.
} 
Quelques années avant qu'Ibn Qunfudh décrive ses expériences dans l'Uns al-faqìr, un autre maghrébin, le voyageur Ibn Bațūṭa, était en train d'entreprendre, avec l'aide du lettré Ibn Juzayy, l'écriture de sa célèbre rị̣la.

Dans les premières pages de celle-ci se trouve un passage qu'il nous semble intéressant d'évoquer ici. Ibn Bațūṭa y décrit son arrivée à Tunis:

«Les habitants de cette ville sortirent à la rencontre du shaykh Abū 'Abdallāh al-Zubaydī et d'Abū Tayyib, fils du qaựi 'Abdallāh al-Nafzawī. Les deux troupes s'approchèrent l'une de l'autre en se saluant et en s'adressant des questions. Quant à moi personne ne me salua, car personne ne me connaissait, ce qui suscita dans mon âme quelque chose qui m'empêcha de retenir mes larmes. Et je me mis à sangloter» ${ }^{27}$.

En apparence, dans ce passage, il n'y a rien de particulièrement remarquable; mais si nous l'examinons de plus près, nous réalisons qu'il s'agit d'un petit morceau assez rare dans le cadre de la littérature musulmane médiévale: les larmes décrites par l'auteur sont cette fois-ci l'expression d'un sentiment de solitude, éprouvé par un individu qui a entrepris un voyage, qui vient de se séparer de sa ville natale et de ses relations familiales et qui, pour la première fois, doit se confronter à la fragilité produite par cette séparation faisant de lui un étranger en chaque endroit. Celui qui écrit est la même personne que celle qui a éprouvé la solitude et qui en a pleuré. Les flots de larmes qu'Ibn Jubayr décrivait deux siècles avant, concernaient toujours d'autres gens, observés par le voyageur, et ces flots de larmes étaient invariablement suscités par le contact avec un lieu saint, ou par l'émotion produite par l'éloquence d'un prédicateur. Quelque chose s'est passé, concernant le statut de l'individu, qui permet à Ibn Batṭutạ, à l'intérieur du genre riḥla, d'écrire ses émotions même si elles ne sont pas des émotions religieuses. Et pourtant il n'y a pas de représentation conceptuelle des états intérieurs: le langage des sentiments et des émotions reste indéterminé. Ce qu'il y a à l'intérieur de l'âme ne peut être exprimé que par le langage du corps.

Si les textes hagiographiques que nous avons évoqués illustrent les modalités médiévales de l'écriture des sentiments et des émotions religieuses, qui accordent au corps un rôle de premier plan, il laissent également transparaître l'opposition fondamentale entre les formes de savoir centrées sur la parole et se transmettant par l'ouïe et celles véhiculées par la vue et les autres sens corporels,

27 Voyages d'Ibn Batoutah, texte arabe, accompagné d'une traduction par C. Defremery et B. R. Sanguinetti, tome I, Paris, 1853. La traduction a été légèrement modifiée. 
impliquant une expérience directe. La parole, instrument privilégié de transmission du savoir éducatif, n'a qu'une place de second plan dans ces récits hagiographiques, tandis que le geste, le contact, le silence y jouent un rôle majeur. Le corps peut véhiculer des connaissances spirituelles qui s'acquièrent par le biais d'un apprentissage informel et la parole, loin d'y tenir une fonction privilégiée, révèle toutes ses limites: certaines expériences ne peuvent se réaliser que dans le silence et par le silence, et les mots n'arrivent pas à les décrire. Les récits hagiographiques mettent en scène cette forme de connaissance, en se mouvant à l'intérieur des frontières invisibles qui circonscrivent l'espace dont l'individu médiéval dispose pour écrire ses émotions.

\section{ABSTRACT}

L'ouvrage de l'hagiographe maghrebin Ibn Qunfudh, Uns al-faqir wa- 'izz al-haqìr, a été choisi comme point de départ d'une recherche concernant les modalités médiévales de l'écriture des sentiments et des émotions religieuses. Le corps nous apparaît dans les récits hagiographiques comme étant le véhicule de connaissances spirituelles et, parmi les sens corporels, la vue, ainsi que le toucher et le goût, ont la prééminence. Une hiérarchie se dessine entre les formes de savoir centrées sur la parole et la transmission auditive et celles, véhiculées par la vue et les autres sens corporels, qui impliquent une expérience de contact direct et qui s'acquièrent par un apprentissage informel où les gestes et les silences jouent un rôle de premier plan.

\section{RESUMEN}

La obra del hagiógrafo magrebí Ibn Qunfud, Uns al-faqir wa-'izz al-haqir, ha sido escogida como punto de partida de una investigación acerca de las modalidades medievales de la escritura de los sentimientos y de las emociones religiosas. El cuerpo se nos aparece en los relatos hagiográficos como vehículo de conocimientos espirituales $y$, entre los sentidos corporales, la vista, el tacto y el gusto tienen preeminencia. Se perfila una jerarquía entre las formas de saber centradas en la palabra y la transmisión auditiva y aquellas, cuyo vehículo son la vista y los otros sentidos, que implican una experiencia de contacto directo y que se adquieren por un aprendizaje informal donde los gestos y los silencios desempeñan un papel protagonista. 